\section{Interacción entre la extensión y la investigación- acción para el abordaje de una problemática en el sector productivo marplatense}

\author{
Mariela Ambrústolo \\ marielaambrustolo@gmail.com
}

Marina Migueles

mmigueles@fi.mdp.edu.ar

María Betina Berardi

bberardi@fi.mdp.edu.ar
RECEPCIÓN: 25/08/18

ACEPTACIÓN FINAL: 28/10/18

\author{
Claudia Zárate \\ cnzarate@fi.mdp.edu.ar \\ Universidad Nacional de Mar del Plata, \\ Argentina.
}

Investigación y extensión universitaria / Intervenciones

\section{Resumen}

El presente escrito tiene por objeto describir el trabajo de investigación-acción del Grupo de investigación y extensión "Mejora Continua, Calidad y Medio Ambiente".

El proyecto se centró en la aplicación de una herramienta básica de gestión que permite preparar a las organizaciones que pretendan implementar sistemas de gestión de la calidad y mejorar sus lugares de trabajo. El mismo fue desarrollado en tres períodos a través de talleres de extensión brindados a empresas representativas del sector industria marplatense y en los cuales se contó con la participación de estudiantes avanzados. La extensión en el ámbito universitario es un desarrollos tecnológicos, capacitación en diversos sectores sociales, productivos públicos o privados. La construcción de conocimiento resultó un elemento fundamental para el desarrollo del proyecto de investigación que abordó el estudio de las modalidades, estrategias, ventajas y obstáculos que se presentan en la aplicación de estas herramientas. mecanismo de construcción de capacidades,
Interaction between extension and action research to address a problem in the Mar del Plata productive sector

\section{Abstract}

The present objective is to describe the actionresearch work of the Research and Extension Group "Continuous Improvement, Quality and Environment".

The project focused on the application of a basic management tool that allows preparing organizations that want to implement quality management systems and improve their workplaces. It was developed in three periods through Extension Workshops for companies that represent the industrial sector of Mar del Plata and where advanced students participated.

Extension in the university field is a mechanism for training, technological development, training in various social sectors, productive public or private. The construction of knowledge was a fundamental element for the development of the research project that addressed the study of the modalities, strategies, advantages and obstacles that arise in the application of these tools.

Keywords: action research, extension, management tools, $5 \mathrm{~s}$.
Interação entre extensão e pesquisa-ação para enfrentar um problema no setor produtivo de Mar del Plata

\section{Resumo}

$\mathrm{O}$ artigo tem o objetivo dedescrever o trabalho de pesquisa-ação do Grupo de Pesquisa e Extensão "Melhoria Contínua, Qualidade e Meio Ambiente". O projeto se concentrou na aplicação de uma ferramenta básica de gerenciamento que permite preparar organizações que pretendem implementar sistemas de gestão da qualidade e melhorar seus locais de trabalho. Foi desenvolvido em três períodos através de Workshops de Extensão para empresas que representam o setor industrial de Mar del Plata e onde participaram estudantes avançados. A extensão no campo universitário é um mecanismo de capacitação, desenvolvimento tecnológico, capacitação em diversos setores sociais, produtivos públicos ou privados. A construção do conhecimento foi um elemento fundamental para o desenvolvimento do projeto de pesquisa que abordou o estudo das modalidades, estratégias, vantagens e obstáculos que surgem da aplicação dessas ferramentas.

Palavras-chave: pesquisa-ação, extensão, ferramentas de gestão, $5 \mathrm{~s}$. Palabras clave: investigación-acción,
extensión, herramientas de gestión, 5s.

\author{
ferramentas de gestão, 5 s.
}




\section{Introducción}

Desde hace algunos años, la actividad de extensión y transferencia ha tomado un rol fundamental dentro de las universidades y se ha revalorizado desde su función. Es señal de esto la relevancia que ha cobrado la extensión en la Universidad Nacional de Mar del Plata (UNMdP) a través de la importancia de las convocatorias para el desarrollo de proyectos y la cantidad de recursos destinados.

En este contexto, el equipo de investigación y extensión "Mejora Continua, Calidad y Medio Ambiente", dependiente del Departamento de Ingeniería Industrial (DII) de la Facultad de Ingeniería (FI) de la UNMdP, hace más de diez años que trabaja en actividades de extensión dentro de la temática de mejora de los procesos, calidad y mejora continua.

Por otra parte, el interés por investigar en estas áreas en función de conocer la situación del sector productivo generó la necesidad de buscar una herramienta de investigación que permitiera establecer una interacción entre las actividades del equipo y un acercamiento mayor a la realidad.

El objetivo del presente trabajo es compartir una intervención desarrollada por el equipo y describir el trabajo de investigación-acción realizado a través de la actividad de extensión.

La selección de la problemática surgió de investigaciones y trabajos anteriores que posibilitaron advertir que era preciso actuar en el ámbito de las organizaciones para la mejora de los espacios de trabajo.

El proyecto se centró en la aplicación de una herramienta básica de gestión que permite preparar a las organizaciones que pretendan implementar sistemas de gestión de la calidad y mejorar prácticas y formas de uso de sus lugares de trabajo para que el trabajador se encuentre en un ambiente seguro y eficaz.

El mismo fue desarrollado a través del Programa Despertar, que involucró a empresas representativas del sector industrial marplatense en tres ediciones. Durante su transcurso, las organizaciones, a través de las actividades, identificaban problemáticas específicas e iban estudiando y desarrollando acciones de mejora con la intervención y el seguimiento del equipo de extensión y estudiantes avanzados de Ingeniería Industrial que habían cursado o estaban cursando la asignatura Gestión de la Calidad. El objetivo del trabajo de investigación fue la construcción de conocimiento a través del desarrollo e implementación de las herramientas y abordó el estudio de las modalidades, estrategias, ventajas y obstáculos que se presentan en su aplicación.

En este artículo se presentarán las perspectivas desde donde se desarrolló el trabajo, la metodología elegida para la intervención, un recorrido por las acciones y herramientas desplegadas en el Programa Despertar, los resultados obtenidos en el ámbito de las instituciones (sector productivo y Universidad) y las experiencias de los estudiantes participantes.

\section{Marco conceptual}

La Universidad es una organización compleja cuyas funciones, objetivos y actividades han ido cambiando a lo largo de la historia para dar respuesta a los nuevos requerimientos internos y externos. Sumado a esto, los fenómenos de la globalización presentan una realidad caracterizada también por una altísima complejidad. Tünnermann Bernheim (2000) expresa que las transformaciones que deberán producirse para lograr el progreso y el desarrollo de la sociedad se fundamentan en el recurso organizacional más importante, que es el conocimiento. 
La misión social de la universidad se ha planteado desde la Reforma de 1918, ramificándose en una serie de actividades diferentes (Frondizi citado por Cecchi et al., 2009). Así, se han planteado cuatro funciones concretas:

"-(...) la formación de profesionales, tanto en su capacidad técnica como en la 'conciencia social'; la misión de '...estudiar los problemas que afligen al país'; en este sentido considera que '...todos nuestros países (refiriéndose a América Latina) sufren males que la universidad puede y debe ayudar a solucionar'.

- (...) la preservación y enriquecimiento de la cultura, que tiene que ver 'con formar profesionales que sepan, disfruten y recreen su saber, lo que implica aprender a pensar, a analizar...'

- (...) el desarrollo de la investigación, tanto en ciencia básica o pura, como en ciencia aplicada o tecnológica

- ayudar a encontrar soluciones para los problemas de desarrollo de un país, a lo que Frondizi denomina 'problemas políticos', función imprescindible si se tiene en cuenta que la Universidad no puede permanecer aislada e indiferente 'frente a una situación que entorpece el progreso del país'". (Cecchi et al::33)

El mismo autor citado define esta mirada de la universidad sobre la sociedad como "Compromiso Social Universitario" y expresa: "La universidad, a través de diversas modalidades y escenarios tiene, concretamente, un diálogo directo con la sociedad, intentando diferentes instancias para lograr su transformación y favoreciendo, fundamentalmente, el logro de la equidad y justicia social" (45).

Camilloni (2010) señala que la universidad constituye un factor estratégico para promover el crecimiento económico, social y cultural y, por consiguiente, el bienestar de la población, y destaca que se encuentra entre las instituciones sociales creadoras de valor. De esta postura emana la capacidad para asumir su misión de formar en valores en sus dispositivos de docencia, investigación y extensión. Pero la realidad es que el gran debate sobre el compromiso social universitario está solamente en sus inicios. Por lo tanto, la profesora propone que se deben asumir nuevos paradigmas: alinear los programas de extensión en función de la visión de la universidad y articular las acciones de extensión con sus otras misiones para contribuir a la solución de problemáticas sociales, entendiendo que la investigación es la que le va a permitir conocer, diagnosticar e identificar las causas de los problemas y programar con eficacia las soluciones. Por otra parte, enfatiza en que la docencia debe formar graduados en condiciones de interactuar efectivamente en su entorno y facilitar la relación teoría-práctica para el abordaje de las problemáticas. Una de sus propuestas avanza sobre la necesidad de desarrollar las actividades de extensión al interior de las carreras de grado y así permitir a la currícula tener un enfoque social y ético.

De este modo, la extensión universitaria resulta ser el camino indicado para hacer investigación como producción del conocimiento y como una herramienta clave para que la universidad se relacione con la comunidad para la resolución de una problemática. Por ello, señala Hernández:

"A través de la extensión, se puede vencer la ruptura entre la universidad y la comunidad ubicada en su área de influencia. Se trata de abrir las puertas de la universidad para que juntas en espacios convencionales o no, se reúnan, intercambien, aprendan una de la 
otra y unidas emprendan los caminos para lograr que el hombre sea sujeto y objeto de los procesos en los cuales participa". (Citado por Chacín, González y Torres, 2007:221)

La extensión universitaria comprende el conjunto de actividades conducentes a identificar los problemas y demandas de la sociedad y su medio, coordinar las correspondientes acciones de transferencia, y reorientar y recrear actividades de docencia e investigación a partir de la interacción con ese contexto. Puesto que es un mecanismo que permite la construcción de capacidades, desarrollos tecnológicos, capacitación en diversos sectores sociales, ya sean productivos públicos o privados, es importante revalorizarlo y generar ámbitos de interacción con las diversas funciones de la universidad. A través de este, queda materializado uno de los compromisos que la universidad tiene con la sociedad en su conjunto: construcción de conocimiento para resolver las problemáticas que la aquejan. Para la intervención objeto de análisis en este trabajo, resultó un elemento fundamental para el desarrollo del proyecto de investigación que se presenta.

La experiencia de trabajar hace más de 10 años en proyectos de extensión hizo posible para el equipo de investigación y extensión "Mejora Continua, Calidad y Medio Ambiente" encontrar un nexo entre esta actividad y la investigación-acción, y es la respuesta lógica que permite conjugar las diferentes funciones que el mismo tiene como objeto a desarrollar.

La intervención que se expone en este trabajo se enmarca en un enfoque de investigación cualitativa con un diseño de investigación acción.

"La finalidad de la investigación-acción es resolver problemas cotidianos e inmediatos (Álvarez-Gayou, 2003; Merriam, 2009) y mejorar prácticas concretas. Su propósito fundamental se centra en aportar información que guíe la toma de decisiones para programas, procesos y reformas estructurales". (Hernández Sampieri et al., 2006:706)

Sandín Esteban señala que la investigación-acción pretende "propiciar el cambio social, transformar la realidad y que las personas tomen conciencia de su papel en ese proceso de transformación" (2003:161) construyendo el conocimiento por medio de la práctica.

La investigación-acción parte de una epistemología pospositivista que propone que las organizaciones son sistemas complejos sobre las cuales no es posible obtener conocimientos significativos mediante la reducción de un proceso determinado a unas cuantas variables o componentes y observar cada una de manera independiente y objetiva sin interferir en las variables estudiadas (Avison, Lau, Myers y Nielsen, 1999). Es por ello que las organizaciones deben estudiarse en forma sistémica, y la mejor estrategia es intervenir en los procesos que se desea estudiar siendo partícipe de las acciones que proponen los actores involucrados a través de las acciones de formación para resolver las problemáticas planteadas, realizar el seguimiento y observar los cambios resultantes.

Dentro de las características más importantes de esta forma de investigar se pueden mencionar las siguientes:

- "La investigación-acción envuelve la transformación y mejora una realidad construyéndose a partir de esta.

- Parte de problemas prácticos que se encuentran vinculados a un determinado entorno.

- Implica la colaboración de los participantes en la detección de necesidades y en la 
implementación de los resultados del estudio ya que ellos son los que mejor conocen la problemática a resolver, la estructura a modificar, los procesos y las prácticas que requieren un cambio". (Sandín Esteban, citado en Hernández Sampieri et al. 2006:707)

Desde otro punto de vista, para muchas problemáticas se requiere un acercamiento gradual y progresivo que permita establecer diferentes etapas de intervención. León y Montero (2002) consideran a la investigación-acción un proceso de pasos "en espiral" donde se investiga y al mismo tiempo se interviene dentro del contexto social.

Alvarez- Gayou (2003) destaca dentro de sus tres enfoques la perspectiva de investigación técnico-científica, en la que se encuadra el trabajo presentado, que parte de la investigación-acción creada por Kurt Lewin y cuyo modelo genera una espiral de ciclos repetidos de análisis que admiten planificar, diagnosticar, implementar acciones y evaluar resultados.

Creswell (citado en Hernández Sampieri et al., 2006) identifica dos diseños fundamentales de la investigación-acción: práctico y participativo. El primero se basa en el estudio de prácticas locales centradas en el desarrollo de los participantes para la implementación de un plan de acción que genere un cambio, la resolución de una problemática y la mejora de una situación. El segundo enfoque estudia temas sociales, busca lograr una colaboración que permita mejorar la calidad de vida y la mejora también de los individuos. Se entiende que la propuesta de esta intervención se alinea con el primer diseño. Para ello, se rescata la "espiral" sucesiva de ciclos que desarrolla Sandín Esteban (2003) y que incluye:

- Identificar el problema de investigación, detectar hechos, analizar y diagnosticar la situación.

- Formular un plan con acciones que permitan resolver el problema y generar un cambio de la situación actual.

- Implementar lo planificado, observar y evaluar resultados.

- Determinar acciones de retroalimentación, que permiten analizar nuevamente la situación generando una nueva espiral de reflexión y acción.

En este camino, el grupo "Mejora Continua, Calidad y Medio Ambiente", dependiente del DIl de la Facultad de Ingeniería de la UNMdP, ha conjugado así las diferentes funciones de la universidad en el Proyecto de Investigación "Estudio del grado de desarrollo y aplicación de las técnicas de gestión de la calidad y ambientales en empresas regionales" con el Taller de extensión "Programa Despertar para organizaciones" y estableció una mecánica de investigación-acción sustentada en las actividades de extensión.

Por otra parte, este concepto de "espiral" mencionado anteriormente y que plantean los autores de mejora sucesiva es el sustento de la filosofía y las herramientas que el equipo de investigación y extensión desarrolla: el ciclo PDCA (Planear, hacer, verificar y actuar por su sigla en inglés: Plan, Do, Check, Act) popularizado por E. Deming y cuya implementación es la metodología usual aplicada en los procesos de cambio.

La otra problemática por resolver cuando se estudia el abordaje de herramientas en el ámbito de los sistemas de gestión es el acceso para poder estudiar los procesos desarrollados. Sin duda, para diferentes ámbitos, el acercamiento a actividades de extensión es más natural y amigable. 
El presente trabajo busca exponer los resultados de esta estrategia para la generación de conocimiento y habilidades en el sector productivo marplatense en el desarrollo de una técnica de gestión que le posibilitará mejorar desde diversos aspectos su realidad actual.

Las herramientas elegidas para el estudio fueron las $5 \mathrm{~s}$, una técnica internacionalmente reconocida que se basa en la mejora del ambiente de trabajo en las organizaciones. El movimiento de la mejora de la calidad comenzó en los sistemas de producción de las empresas japonesas de automoción, cuando empezaron a utilizar estrategias, métodos y herramientas de mejora que les proporcionaron una forma de trabajar que permitía mantener una producción flexible, aumentando la calidad con enorme ahorro de costes. Así, surgió el Kaizen, que proviene de la unión de dos vocablos japoneses: $K A l$, que significa cambio, y ZEN, que quiere decir bueno o bondad. Tal como lo describe Imai:

"Mejoramiento progresivo que involucra a todos, incluyendo tanto a los gerentes como a los trabajadores. La filosofía de Kaisen supone que nuestra forma de vida — sea nuestra vida de trabajo, vida social o vida familiar— merece ser mejorada de manera constante". (2001:39)

Dentro de esta filosofía surgen las $5 \mathrm{~s}$, que son herramientas de la calidad nacidas en Japón después de la Segunda Guerra Mundial con el fin de descubrir mejores formas de hacer las tareas y facilitar el trabajo. El objetivo principal es eliminar desperdicios, hacer el trabajo más productivo y confortable, mejorar la calidad y el lugar de trabajo.

Imai desarrolla los cinco pasos del housekeeping, con sus nombres japoneses, como sigue:

"1. Seiri: diferenciar entre elementos necesarios e innecesarios en el lugar de trabajo y disponer estos últimos.

2. Seiton: disponer en forma ordenada todos los elementos que quedan después del seiri.

3. Seiso: mantener limpias las máquinas y los ambientes de trabajo.

4. Seiketsu: extender hacia uno mismo el concepto de limpieza y practicar continuamente los tres pasos anteriores logrando la estandarización de las prácticas.

5. Shitsuke: construir autodisciplina y formar el hábito de comprometerse en las $5 \mathrm{~s}$ mediante el establecimiento de estándares". (1998:Cap. 5, 1)

Dorbessan (2001) resalta que es una herramienta que permite producir un cambio en el cual, a medida que se van implementando las tres primeras acciones, que se realizan en forma grupal, participativa y autónoma, los miembros del grupo incorporan este método por los beneficios que obtienen. Un hábitat laboral agradable, limpio y ordenado, que trae beneficios directos tales como mejorar la calidad, productividad y seguridad, entre otros. El aprendizaje de trabajar grupalmente rescata los conocimientos de las personas adquiridos en su accionar, convierte a la organización en organización de aprendizaje y crea las condiciones para aplicar modernas técnicas de gestión.

La estrategia de las $5 \mathrm{~s}$ es una buena herramienta básica para las empresas que quieren ordenarse y comenzar a empezar a aplicar los primeros conceptos de calidad

Imai (2001) describe que las 5s es un programa de participación expandida en las compañías que incluye a todo el personal de la organización. Tiene un alcance muy efectivo para motivar a la gente y mejorar el ambiente de trabajo, la productividad y la eficiencia.

A su vez, incorpora varios conceptos importantes que luego servirán de base para la puesta en funciones de modelos más avanzados, como lo son los sistemas de gestión de la 
calidad. En la implementación de las 5s se generan aprendizajes que permiten incorporar el liderazgo, la participación, el trabajo en equipo, la filosofía de la mejora continua, y se trabaja con la resistencia al cambio, factores fundamentales que se capitalizarán para una futura utilización de un sistema de gestión, por lo que resulta una herramienta básica imprescindible para el comienzo del camino hacia la calidad. Por todo esto, es un tema central de estudio del equipo de investigación y extensión.

\section{Metodología de trabajo}

El estudio tuvo sus orígenes durante los años 2010 y 2011, a partir del proyecto de investigación "Estudio del grado de desarrollo y aplicación de las técnicas de gestión de la calidad y ambiental en empresas regionales". En dicho proyecto se abordó el estudio de casos de empresas de la ciudad de Mar del Plata que poseían un sistema de gestión de la calidad basado en la norma internacional ISO 9001. El objetivo del proyecto era conocer cómo abordaban la mejora continua las empresas, sus beneficios e inconvenientes, con el fin de identificar oportunidades de mejora y dar respuesta a las necesidades del sector empresario como función de la universidad.

Como resultado de ese análisis se encontraron conclusiones no esperadas a partir de las proposiciones establecidas al iniciar el estudio.

Dentro de ellas, una de las primeras fue que se presumía que el principal motivo de la implementación del sistema de gestión de la calidad fuera de carácter externo, de manera de aumentar la competitividad en los mercados donde las empresas comercializaban sus productos. Cruz Ríos, Ubeda García y Limañasa Gresa (2007) señalan en motivos externos, de mercado o entorno (exigencias de clientes y/o mercados, aumentar la competitividad internacional, adquirir nuevos mercados, presión por la competencia, mejorar la imagen, etc.), los motivos internos (mejorar la calidad del producto/servicio, meta establecida por la dirección, mejorar la organización y la gestión, etc.), y motivos de eficiencia (asociados a la reducción de costos y satisfacción del personal).

En la misma línea, trabajos desarrollados por Formento, Braidot y Pittaluga (2007) indican que el sector industrial argentino, en los últimos años y en un escenario de constantes transformaciones económicas, se ha visto impulsado a la inserción en un contexto internacional regido por la globalización. Sin embargo, del estudio de casos (Ambrústolo, Migueles, y Pascual, 2012) se determinó que, dentro de los principales beneficios de la implementación de la norma ISO 9001, las empresas marplatenses señalaban:

- Ordenar y sistematizar los procesos de la organización.

- Lograr el cumplimiento de los objetivos.

- Crecimiento continuo.

- Controles periódicos apoyados en un sistema documental.

Esto hizo posible determinar que las empresas no utilizaban en forma preventiva las herramientas de la mejora continua y desperdiciaban la potencialidad de las mismas. Por otra parte, estaban más enfocadas en una motivación interna para generar un orden y un mejoramiento del trabajo. 
A partir de esta situación, el grupo de investigación y extensión se planteó la necesidad de la intervención por parte de la Universidad en estas cuestiones y, a partir de los resultados del proyecto, se estableció un nuevo programa de investigación-acción que, combinado con actividades de extensión, permitiera dar una respuesta a las problemáticas identificadas.

Para ello, se eligió el Programa Despertar, desarrollado hacía varios años por el mismo equipo, basado en las técnicas internacionales 5 s para actividades de investigación y extensión que les faciliten a las empresas conocer herramientas básicas de organización e incorporar conceptos de prevención y metodológicos con el objeto de ser el puntapié para la aplicación de herramientas de calidad más avanzadas. Las 5 s son indicadas para lograr esta base sólida de conocimientos y habilidades y para trabajar en valores necesarios para cualquier herramienta de calidad: aprendizaje, trabajo en equipo, teoría del cambio, mejora continua y adecuación permanente.

En tanto, la única forma de comprender la potencialidad de estos modelos, sus dificultades y ventajas en las organizaciones, es a partir de la intervención y el seguimiento de la implementación in situ.

Es por ello que durante seis años se trabajó en el proyecto de investigación cualitativa combinando la metodología de estudio de casos con investigación-acción, articulándolos con actividades de extensión para tener una clara presencia en todo el desarrollo del programa.

En el primer período de trabajo (2012/2014) se establecieron las metodologías y se realizó un intento de desarrollar investigación-acción a través del Programa Despertar. Si bien este primer intento no tuvo los resultados esperados, de la experiencia surgieron las bases para mejorar metodológicamente el desarrollo del proyecto y poder implementar dos ciclos con éxito. En las Ediciones $2(2014 / 15)$ y $3(2016 / 7)$ se pudieron completar todas las actividades con la participación de siete estudiantes avanzados de Ingeniería Industrial que realizaron su Práctica Profesional Supervisada (PPS) en el proyecto.

El objetivo del trabajo fue detectar inconvenientes en la implementación de estas herramientas e inquietudes del sector con el fin de generar un diagnóstico para:

- Conocer el estado actual respecto de las prácticas básicas de calidad.

- Concientizar y capacitar a las empresas del estudio para la implementación de las técnicas $5 \mathrm{~s}$.

- Establecer fortalezas, oportunidades, debilidades y amenazas en la implementación de las últimas etapas de las 5 s en empresas regionales.

- Determinar factores de éxito que permitan dar continuidad y permanencia al Programa.

- Formar el trabajo profesional supervisado en los estudiantes a través de las PPS.

- Proporcionar información necesaria para que la Universidad pueda colaborar con el sector productivo.

Para el desarrollo del proyecto se plantearon las siguientes actividades:

- Selección de las preguntas del estudio en función de los objetivos particulares del proyecto y que puedan dar respuesta a los objetivos generales.

- Revisión bibliográfica para establecer las mejores prácticas de investigación cualitativa y de extensión.

- Determinación de las proposiciones que podrían dar respuesta a las preguntas de in- 
vestigación y que permitir desplazarse en la dirección de la búsqueda de evidencias así como el desarrollo de estrategias.

- Detección y definición del problema de investigación.

- Definición de la posible unidad de análisis.

- Desarrollo del Protocolo de trabajo, que contiene los instrumentos, procedimientos y reglas generales para llevar a cabo el estudio en cuestión.

- Selección de la herramienta de interacción con la unidad de estudio.

- Formulación de un plan o programa para abordar y resolver el problema e introducir el cambio.

- Implementación del plan o programa.

- Colección de los datos a través de la búsqueda de múltiples fuentes de evidencias.

- Evaluación de los resultados (determinación de criterios y categorías y análisis cuanticualitativos).

- Retroalimentación, mejora del diagnóstico y definición de una nueva espiral de reflexión y acción.

- Elaboración del informe final.

El diagnóstico fue desarrollado a través de los proyectos de investigación anteriores que dieron origen al presente trabajo y del contacto con el sector productivo, mediante las actividades de docencia y otras acciones que lleva adelante el equipo.

La metodología de interacción que se identifica como actividad disparadora consistió en talleres de capacitación sobre las herramientas 5s: Programa Despertar para organizaciones.

La unidad de análisis fue definida a través del grupo de empresas que voluntariamente participaron en los tres períodos de este Programa y que tienen contacto con la facultad a partir del desarrollo de PPS, actividades de capacitación o diferentes encuentros.

Para la investigación se diseñaron diferentes elementos que permitieron obtener información a lo largo de todo el proceso. La metodología de trabajo contempló la autoevaluación a través de Check list iniciales por parte de las organizaciones intervinientes, la realización de un taller teórico-práctico con la obligatoriedad de un plan de implementación que se fue desarrollando en los diversos encuentros, seguimiento por medio de visitas y entrevistas utilizando observación directa, registro fotográfico, posevaluación por Check list por parte de las organizaciones y por el equipo de extensión, y entrevistas.

El taller se planificó con el objetivo de iniciar a las empresas en las herramientas básicas de la calidad 5 s y luego realizar el seguimiento de la implementación en los sectores piloto elegidos por las organizaciones a través de las diferentes herramientas antes mencionadas y la participación de estudiantes avanzados en todo el desarrollo. Allí, los participantes podrían comprender e integrar en su desempeño laboral y personal los principios y herramientas de las $5 \mathrm{~s}$, mejorar su disciplina y motivación para lograr un ambiente de trabajo ordenado y eficiente y mejorar la productividad. Fue desarrollado durante tres ciclos, en 2012-2013, 2014-2015 y 2016-2017, en varios encuentros, en el aula magna de la Facultad de Ingeniería, y luego fue monitoreado mediante visitas que permitieron observar la implementación, tener contacto con todos los actores, e ir pensando en conjunto acciones para la resolución de las problemáticas. Aquellos que cumplieran las etapas planificadas del Programa, obtendrían el certificado de aprobación. 
El objetivo fue que las empresas implementaran la herramienta en un sector piloto y que, desde el equipo, se realizara, con la participación y seguimiento de la implementación, un estudio de investigación para determinar estrategias, ventajas y obstáculos. El seguimiento se hizo a través de estudiantes avanzados de Ingeniería Industrial que efectuaron su PPS con la supervisión y el acompañamiento docente.

Por último, luego de establecido un tiempo de implementación, en la tercera edición, se programó una jornada de puesta en común de las actividades realizadas que posibilitó a las diferentes organizaciones compartir sus experiencias y establecer estrategias comunes, dificultades, resultados obtenidos y ventajas. Este encuentro generó autoevaluación y evaluación de todo el Programa y se sentaron bases para la mejora en la aplicación de estas herramientas tanto para las organizaciones como para el mismo Programa. Este espacio permitió a las empresas repensar sus prácticas, compartir con otras organizaciones con similares y diferentes problemáticas y planificar un sostenimiento efectivo del programa generando una espiral de mejora que redundará en la eficiencia del mismo.

Una vez cumplimentadas las actividades participativas, los estudiantes avanzados, junto al equipo de investigación, realizaron informes donde documentaron todos los pasos del plan en cada organización. También se llevaron a cabo reuniones de seguimiento y conclusión para el análisis de los resultados. A partir de estas reuniones, se identificaron acciones que fueron mejorando las actividades del propio proyecto o establecieron las bases de mejora para el ciclo siguiente, creando la "espiral" de reflexión y acción.

\section{Actividades y resultado del Programa Despertar}

\section{Principales actividades desarrolladas}

Una de las misiones del equipo de investigación "Mejora Continua, Calidad y Medio Ambiente" es difundir y afianzar herramientas que permitan la mejora continua en la gestión de las organizaciones. Es por ello que con las actividades se intenta tener una línea de continuidad es lo para generar un mayor conocimiento del entramado productivo.

A continuación, se describirá y analizará el abordaje del Programa Despertar y todas las actividades de investigación y extensión desarrolladas.

El primer paso para la implementación del Programa, como se mencionó en el apartado anterior, fue el desarrollo del diagnóstico. El mismo estuvo basado en proyectos de investigación precedentes que determinaron las siguientes necesidades básicas en las organizaciones casos (Ambrústolo et al., 2012):

- Orden y organización.

- Involucramiento de los directivos.

- Compromiso del personal.

- Sistematización de la mejora.

- Implementación de metodologías sencillas.

- Enfoque preventivo.

El segundo paso para la puesta en práctica de un mecanismo de investigación-acción consistió en desarrollar la estrategia participativa para llevarla a cabo. Como se mencionó 
en el apartado de metodología, el instrumento elegido fue el taller de extensión "Programa Despertar para las organizaciones". El mismo fue aprobado por el Consejo Académico de la Facultad de Ingeniería.

Para esta la estrategia se trabajó en dos aspectos fundamentales: el taller con actividades didácticas y la selección de diversas puertas de entrada al conocimiento. Se desarrollaron juegos, casos de estudio y actividades de puesta en común entre los participantes de modo de enriquecer el aprendizaje. Las diferentes actividades plantearon desafíos a las organizaciones y crearon espacios para planificar e implementar las herramientas objeto de estudio en un área piloto. El segundo aspecto fue el referente a los materiales de apoyo para registrar los diagnósticos particulares y los avances realizados por las organizaciones a través de todo el Programa. Aquí se trabajó con técnicas específicas de las herramientas 5s y con técnicas de investigación.

En primer lugar, se utilizó una lista de chequeo para establecer un autodiagnóstico respecto de la situación inicial de la organización antes de comenzar la aplicación de las herramientas. Se presenta la primera página a modo de muestra en la Figura 1.

Figura 1. Check list Programa Despertar.

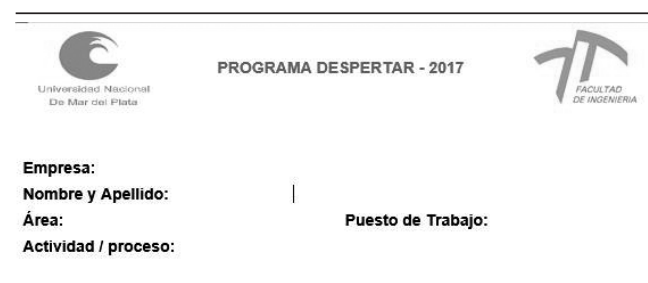

Puntaie representativo:
Muy alto (100 - 80), Alto (80 - 60), Medio (60 - 40), Bajo (40 - 20), Muy Bajo (20 - 0).

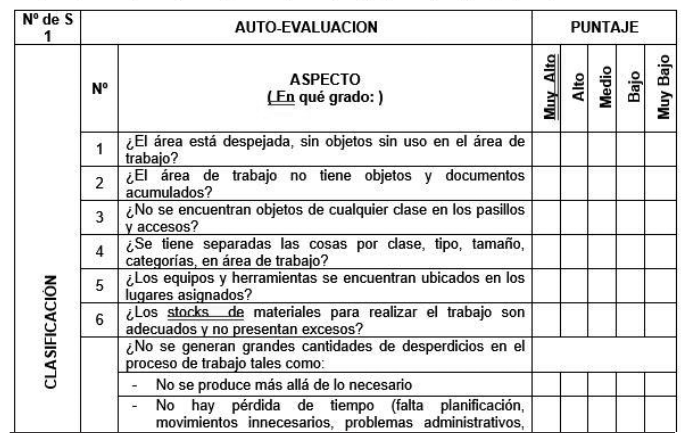

Fuente: elaboración propia (2010).
Figura 2. Gráfico Radar Programa Despertar.

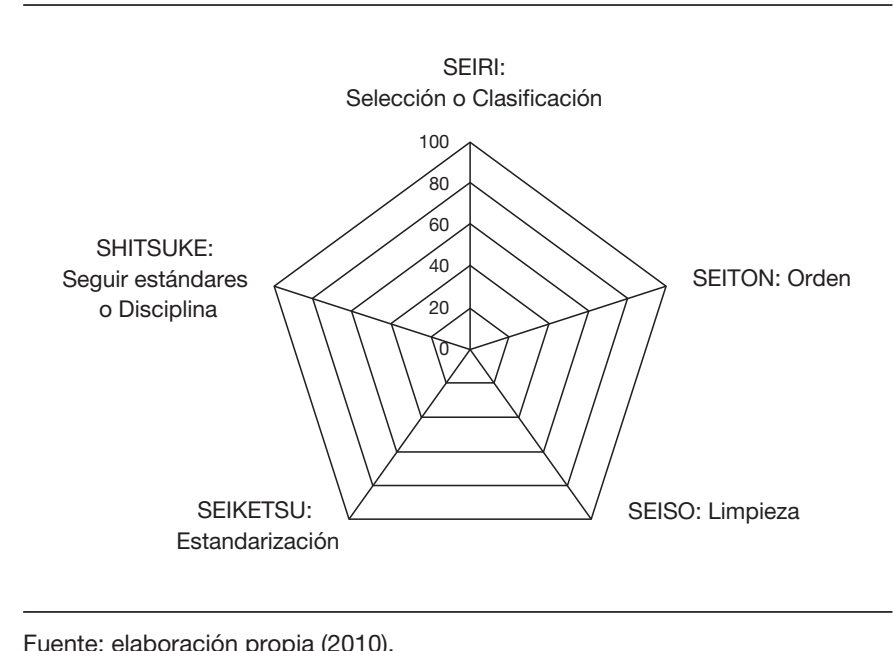

Fuente: elaboración propia (2010).

Los resultados obtenidos se plasman en un gráfico radar (Figura 2) que se convierte en una herramienta visual que permite realizar los sucesivos seguimientos y establecer los avances logrados con la herramienta implementada. En el mismo se grafica el resultado obtenido en la evaluación por cada "s" y se traza una línea común.

A su vez, se apoya el diagnóstico con material fotográfico para tener una visión de la situación inicial. Esta estrategia genera compromiso en los participantes y evidencia las mejoras cuando, al concluir la implementación, se compara con la situación final del ambiente de trabajo. Un ejemplo del registro se muestra en la Figura 3. 
Figura 3. Registro fotográfico Programa Despertar.

Plan de implementación - Registro

\begin{tabular}{|c|c|c|}
\hline \multicolumn{2}{|l|}{ Acción: } & Nro. de S: \\
\hline Grupo: & Líder: & Responsable: \\
\hline
\end{tabular}

Fotografía de antes de comenzar la acción; Fecha: .../.../...

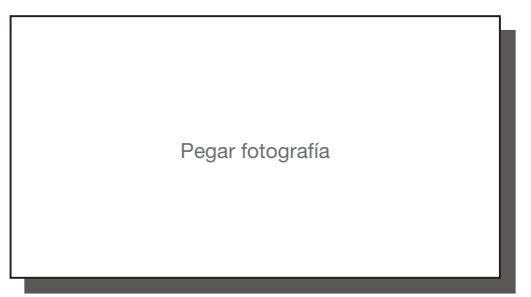

Fotografía de después de finalizar la acción; Fecha: ......./...

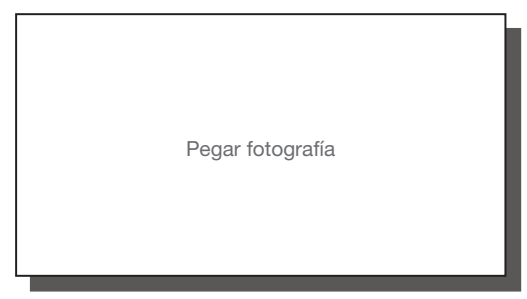

Fuente: Dorbessan (2001).

Si bien estas fueron las actividades iniciales, a lo largo del taller se fueron desplegando y diseñando otras que posibilitaron establecer la aplicación de las $5 \mathrm{~s}$. En la Figura 4 se muestra una tabla que resume la planificación de las actividades en el taller.

Figura 4. Gráfico Radar Programa Despertar.

\begin{tabular}{|c|c|c|c|c|}
\hline & Acciones & Responsables & Tiempo & Recursos \\
\hline \multicolumn{5}{|c|}{ Realizar autodiagnóstico. } \\
\hline \multicolumn{5}{|c|}{$\begin{array}{l}\text { Determinar innecesarios y planificar su eliminación. } \\
\text { Usar etiquetas rojas. }\end{array}$} \\
\hline \multicolumn{5}{|c|}{ Hacer croquis. Aplicar orden. } \\
\hline \multicolumn{5}{|c|}{$\begin{array}{l}\text { Definir el día de las } 5 \mathrm{~s} . \\
\text { Establecer acciones de limpieza. necesarios. Armar } \\
\text { Check list. Usar etiqueta amarilla. }\end{array}$} \\
\hline \multicolumn{5}{|c|}{ Aplicar gestión visual y estandarización. } \\
\hline $\begin{array}{l}\text { Planificar cartelera. } \\
\text { Planificar auditoría } 5 \text { s. }\end{array}$ & & & & \\
\hline
\end{tabular}

Fuente: elaboración propia (2010).

Todo el material recopilado con los diferentes instrumentos fue compilado para efectuar un análisis posterior. Para esta actividad se asignó como responsable a un estudiante avanzado que debía realizar el seguimiento y registro de tres organizaciones intervinientes. 
Al finalizar el taller, se articuló una encuesta a cada organización para reflexionar sobre los avances realizados y establecer acciones de mejora. La misma incluía los siguientes aspectos: grado de desarrollo de cada "s", metodología de implementación aplicada en cada caso, percepción del grado de mejora obtenido con el Programa Despertar, consecuencias logradas con la aplicación de las $5 \mathrm{~s}$, impacto de las mejoras obtenidas, evaluación del compromiso de todos los niveles, herramientas planificadas para dar continuidad al Programa dentro de la organización, inconvenientes encontrados en las diferentes fases y evaluación general. Toda esta información fue cruzada con los resultados obtenidos a través de observación directa en las visitas y de las entrevistas a los participantes del Programa.

\section{Resultados del Programa Despertar para las organizaciones}

A la primera edición del taller asistieron 17 personas de diferentes empresas e instituciones; la asistencia no era obligatoria, ni un requisito del desarrollo de las herramientas en un sector piloto para la entrega de los certificados. Estos factores influenciaron para que las organizaciones aprovecharan el taller pero no derivaron en la aplicación de las herramientas y no permitieron el seguimiento de las mismas. Al no realizar la implementación, tampoco se logró verificar la potencia de estas herramientas ni producir un cambio en las organizaciones. El único resultado importante de esta edición fue que despertó la iniciativa de una empresa en implementar el Programa a través de la solicitud de un curso in-company. Si bien este curso se alejaba del objetivo del Programa, permitió al equipo probar y validar las herramientas de capacitación y seguimiento desarrolladas en un ambiente acotado.

En el segundo taller participaron 18 empresas y 2 áreas internas de la FI, en su mayoría PyMEs. El personal que participó fue variado respecto del tipo de trabajo y cargo dentro de la organización, pero cada participante debió involucrar a su grupo de trabajo para la implementación del Programa.

En la Figura 5 puede observarse el autodiagnóstico que refleja el estado inicial antes de la implementación de las $5 \mathrm{~s}$ en las empresas, donde el promedio está representado por un cuadrado con sus desviaciones máximas y mínimas. Luego, el cursor romboidal muestra los promedios obtenidos por las organizaciones a partir de la implementación del Programa y sus desviaciones a través de triángulos. Se destaca un gran avance en las primeras herramientas y un crecimiento más conservador en el resto. Esto se debe a que las mismas requieren de mayor tiempo para la implementación y un mayor involucramiento en la cultura organizacional.

A pesar de que el $60 \%$ de las empresas posee un sistema de gestión de la calidad, no se aprecia un alto nivel de estandarización y disciplina excepto en una de ellas, que cuenta con un completo sistema documental y genera un punto máximo de estandarización elevado.

En esta edición, de 20 organizaciones que comenzaron el Programa, cuatro desertaron y no completaron el taller. La totalidad de las restantes realizó el autodiagnóstico, y un $69 \%$ de ellas completó el curso e hizo el seguimiento. El $44 \%$ de las entidades participantes implementó efectivamente el Programa, con un $61 \%$ de asistentes al curso. Se evidenció que, cuando fue el grupo de trabajo de la organización el que participó del taller y no solamente un representante, los resultados fueron más positivos.

En la tercera edición del Programa Despertar, lo cumplieron 28 personas de 10 empresas y 1 proyecto interno de la Fl con características similares a la primera edición. 
Figura 5. Comparación de los estados inicial y posterior a la implementación del Programa.

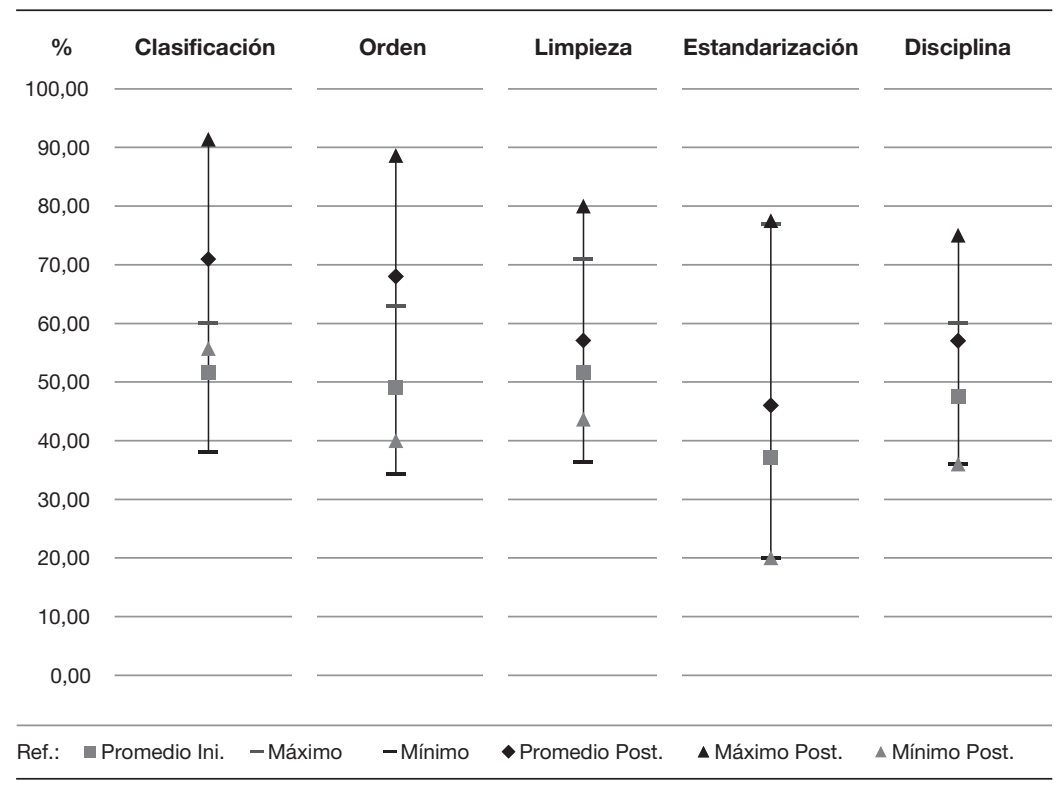

Fuente: Programa Despertar Edición 2. Elaboración propia.

Figura 6. Estado inicial de la evaluación del Programa Despertar Edición 3.

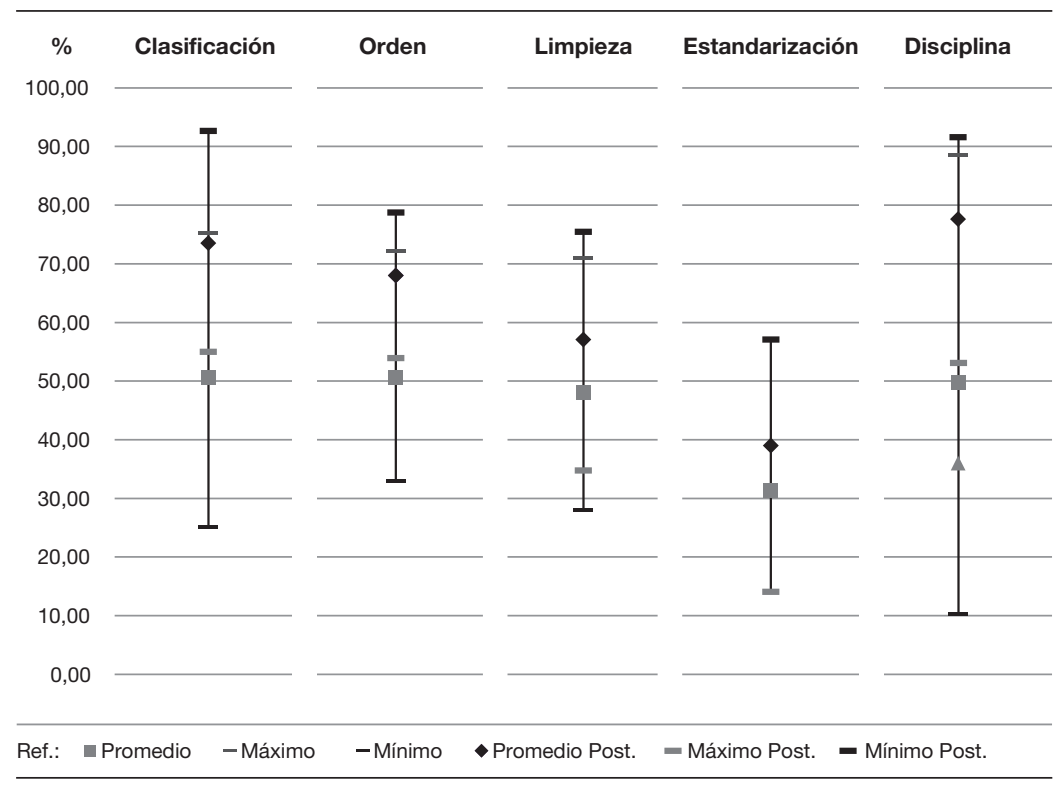

Fuente: Elaboración propia.

En la Figura 6 puede observarse la situación inicial de las organizaciones representadas por un cuadrado, donde presentan niveles cercanos y menores al $50 \%$ respecto de la caracterización de los lugares de trabajo, aún menores que en la edición anterior aunque con 
una mayor dispersión. Luego, el cursor romboidal muestra los promedios parciales obtenidos por una parte de la muestra (63 \%) ya que hay un grupo de organizaciones que no pudo completar la visita final. Como en el caso de la primera edición, se destaca un mayor avance en las primeras herramientas y un crecimiento más conservador en la estandarización por el tipo de procesos involucrados, pero, sin duda, un gran mejoramiento de la herramienta "disciplina". Esto se debe a que varias de las organizaciones poseían estándares de trabajo que permitieron, junto al desarrollo de las actividades del taller, lograr un gran involucramiento del personal y generar nuevas formas de trabajo.

De todas formas, a partir de la primera experiencia y del seguimiento de lo ya realizado en la segunda edición, se observa que un gran porcentaje de las empresas comprendió las herramientas enseñadas, generó propias estrategias de implementación y adaptó las mismas a sus necesidades.

Una de las mejoras establecidas en la Edición 3 fue la actividad final que, como se mencionó, tuvo el objetivo de abordar la problemática de la implementación dispar de las diferentes s. En la misma se pusieron en común estrategias y modalidades de las diferentes organizaciones y se trabajó fuertemente sobre la importancia de generar la disciplina para el sostenimiento del Programa.

En esta instancia, se realizó una encuesta de evaluación del taller y una autoevaluación de la forma de trabajo y de la implementación del Programa Despertar por parte de las organizaciones. Es importante mencionar entre los resultados de dicha encuesta que el $80 \%$ destacó que la implementación de las 5 s tuvo un impacto alto y muy alto respecto de la mejora del lugar del trabajo para la organización y el $100 \%$ manifestó que ese impacto fue alto y muy alto para el equipo de trabajo que llevó a cabo el desarrollo de las actividades del Programa Despertar. Las mayores ventajas que resaltaron los equipos tuvieron que ver con la liberación de espacios en los sectores de trabajo y, luego, como ventajas secundarias, señalaron la disminución de tiempos, costos, mejora en la imagen y la habitabilidad de los espacios.

En cuanto al taller de extensión, el $80 \%$ de las organizaciones apuntó que tuvieron una alta y muy alta comprensión de todos los involucrados en el proyecto 5 s y que el Programa Despertar generó un alto y muy alto compromiso en el desarrollo de las tareas, lo cual es un factor primordial para producir cambios organizacionales. Esto llevó a que las empresas puntuaran al taller de extensión con 8,9 y 10 puntos en una escala de 1 a 10 , hecho que permite evaluar en forma global las actividades desarrolladas por el equipo de extensión.

Por último, más allá de los resultados del taller, es importante hacer hincapié en que las acciones logradas no solo apuntan a mejorar las actividades de las organizaciones sino a lograr lugares de trabajo más cómodos y seguros.

\section{Resultados del Programa Despertar para el equipo de investigación y extensión}

En cuanto a los resultados evaluados desde el punto de vista de los objetivos planteados, mediante el Programa Despertar basado en 5 s se pudieron identificar los diferentes factores de éxito e inconvenientes que tienen las empresas al momento de implementar estas herramientas básicas.

Por otra parte, una de las primeras dificultades se evidenció en la primera edición del taller; si bien metodológicamente se había estudiado la forma de llevar a cabo la experiencia, no se pudo lograr el compromiso de las empresas para trabajar juntas en el seguimiento y 
aplicación de las herramientas. Esta experiencia provocó un replanteo en las estrategias de investigación y comunicación que permitieron plantear un contrato simbólico de cooperación entre los diferentes actores.

El hecho de establecer la obligatoriedad del seguimiento para la entrega de los certificados no solo posibilitó que el programa fuera posible sino que generó el compromiso y la necesidad de la elección de un sector piloto para la implementación. De esta manera, lograron pasar de la mera generación de conocimientos a lo largo de un taller de extensión al desarrollo de habilidades dentro de la organización.

Esta posibilidad está centrada en la espiral de reflexión y retroalimentación que permite este tipo de investigación. Esta estrategia se probó en la segunda edición y resultó muy efectiva.

A su vez, es importante destacar que se pudo planear en cada organización participante esta espiral de reflexión y retroalimentación a partir de las diferentes actividades propuestas y de la secuencia de visitas que originó el replanteo de acciones sobre el diagnóstico y sobre cada aprendizaje logrado.

Sin embargo, como los avances que tuvieron las primeras 3 s respecto de las últimas fue dispar, se introdujeron mejoras al Programa. Esta cuestión surge de la evaluación de la segunda edición y permite un replanteo de estrategias y la incorporación de otras actividades para superar este inconveniente. Como se observa en la Figura 6, se produjeron resultados muy positivos en el desarrollo de la quinta "s" pero no se logró un gran avance en la cuarta. Si bien por el tipo de procesos se preveía este tipo de resultados, se esperaba un avance mayor. De todas maneras, esta situación ayudó al equipo de extensión a estudiar la dinámica de las organizaciones y a establecer las mayores dificultades en cada paso de la implementación de estas herramientas con el objeto de explicitarlas y generar un pensamiento en conjunto sobre estas problemáticas.

Con relación a las fortalezas, es importante resaltar el desarrollo de una estrategia de intervención efectiva que hace factible la aplicación de la investigación-acción centrada en las actividades de extensión donde se permite que los destinatarios participen de una manera natural de las mismas y se involucren en el desarrollo de las propias habilidades e ideas.

En otro aspecto, todo el programa posibilitó ir probando, mejorando y validando herramientas de seguimiento y registrar datos fundamentales para el objeto de estudio.

Finalmente, la incorporación de estudiantes avanzados mejoró el seguimiento del Programa y brindó un espacio más para las actividades de docencia. Cabe destacar que todo el trabajo con los estudiantes impulsó que estas temáticas se profundizaran en la asignatura Gestión de la Calidad a través de la presentación de casos reales.

Integrar la extensión y la docencia a la investigación generó una sinergia de retroalimentación de estrategias y beneficios que dio muy buenos resultados en el Programa y permitirá seguir la línea de trabajo mediante el abordaje de otras herramientas que tiendan a la mejora del sector productivo marplatense.

\section{Resultados del Programa Despertar para los estudiantes avanzados}

Otra cuestión a analizar es la participación de los estudiantes avanzados en la experiencia de de los proyectos llevados a cabo. Las mismas fueron extractadas de los informes entregados al supervisor de PPS. 
La mayoría de los estudiantes destaca las principales ventajas al realizar su práctica en este tipo de proyectos:

- Conocimiento de diversidad de empresas, instituciones, procesos y sus diferentes realidades.

- Relación con profesionales, contacto con los actores directos y sus necesidades.

- Posibilidad de profundizar y consolidar herramientas así como de generar propuestas de mejora.

- Intercambio y retroalimentación entre el grupo de investigación, los pasantes y las empresas en un trabajo en equipo.

- Desarrollo de las habilidades de observación y comunicación.

- Evidencia de las amplias posibilidades laborales de un ingeniero industrial, en las que pueden visualizarse los diversos escenarios a los cuales transferir los conocimientos.

Dentro de los aspectos negativos se identificaron los siguientes:

- Desmotivación generada por las dificultades que se les presentaron.

- Necesidad de adaptación a los tiempos de las diferentes organizaciones.

- Inconformidad debido a que no todas las propuestas pudieron ser implementadas.

- Tiempo de duración de la práctica, lo cual les impidió observar todo el proceso y los resultados de los proyectos en los que fueron partícipes.

Sin embargo, el abordaje de estas situaciones también constituyó un aprendizaje valioso para su futuro desempeño profesional y son las dificultades que normalmente surgen en este tipo de proyectos donde se produce una intervención y no solo una mera capacitación.

Respecto de las competencias desarrolladas, los estudiantes concluyeron casi en forma unánime que su participación en los proyectos les permitió afianzar la responsabilidad y compromiso, la autonomía, el poder de observación, la capacidad analítica, la comunicación oral y escrita y la capacidad para trabajar en equipo.

\section{Conclusiones}

El presente trabajo condensa un largo trabajo del equipo de investigación y extensión y hace posible una reflexión cronológica y global de todas las actividades desarrolladas a través del Programa Despertar para las organizaciones.

Permitió difundir en el sector productivo la necesidad de la incorporación de herramientas para mejorar sus procesos, su eficiencia y productividad, crear lazos con las organizaciones y una demanda compartida en la necesidad de seguir trabajando, investigando y promoviendo mejoras en las formas de trabajo y de asociación con la Universidad, canales que, sin duda, son un desafío pero también un compromiso en la superación de objetivos.

El desarrollo del Programa Despertar produjo la unión de las necesidades de investigación de los equipos de docentes con dedicación exclusiva con la motivación de resolver problemáticas reales interviniendo directamente en la construcción de nuevas formas de trabajo.

Desde el aspecto metodológico, posibilitó al equipo crecer y mejorar todas las herramientas desarrolladas y aplicadas, estableciendo un camino de trabajo aplicable a diferentes 
temáticas. La metodología de investigación acción con un disparador importante, como fue el taller de extensión, gestó una mecánica de trabajo donde se pudo poner en práctica la espiral de reflexión y retroalimentación necesaria para la generación de habilidades. Esto hace que los actores participantes luego puedan replicar estas metodologías de trabajo abordando otras problemáticas sin la dependencia de los integrantes del equipo de extensión, lo cual es uno de los principales objetivos de la producción de conocimientos.

Y, por último, cabe destacar la mejora de la enseñanza de los estudiantes que serán los futuros profesionales, puesto que la participación en este tipo de proyectos les permite acceder a una mayor preparación y mejores condiciones de inserción en la sociedad, proveyéndoles una fuerte experiencia práctica de muy difícil reproducción en el aula.

\section{Referencias bibliográficas}

Alvarez- Gayou, J.L. (2003). Como hacer investigación cualitativa: fundamentos y metodología. México: Paidós. Ambrústolo, M.; Migueles, M. y Pascual, B. (2012). Estudio De Casos: Mejora Continua En Empresas Marplatenses. COINI 2012 Vº Congreso Argentino de Ingeniería Industrial. Tomo 1, 19

Avison, D.; Lau, F.; Myers, M. \& Nielsen, P.A. (1999). Action Research. Communications of the ACM, 42(1), 94-97. Camilloni, A. (noviembre de 2010). Calidad educativa y compromiso social. Desafíos de la extensión. Calidad Educativa y Compromiso social. Panel Inaugural Ilevado a cabo en el IV Congreso de Nacional de Extensión Universitaria - IX Jornadas Nacionales de Extensión Universitarias. Mendoza.

Cecchi, N.; Lakonich, J.; Pérez, D. y Rotstein, A. (2009). El Compromiso Social de la Universidad Latinoamericana del Siglo XXI. Entre el debate y la acción. Mar del Plata: IEC CANADU -Ediciones Suarez.

Chacín, B.; González, M. y Torres, Y. (2007). Crítica a la Generación del Conocimiento en la Extensión Universitaria: Aproximación a un protocolo de investigación innovativa. Laurus, 13(24), 215-240. Recuperado de http://www.redalyc.org/pdf/761/76111485011.pdf

Cruz Ríos, R.; Ubeda García, J.; Limañasa Gresa, J. (2007). Principales motivos que conducen a la implantación de un sistema de gestión de la calidad y principios que subyacen. Decisiones basadas en el conocimiento y en el papel social de la empresa: XX Congreso anual de AEDEM, 1, 37.

Dorbessan J. (2001). Las 5S, herramientas de cambio. Buenos Aires: Editorial de la UTN.

Formento, N.; Braidot, P. y Pittaluga, J. (2007). El proceso de mejora continua en PyMEs Argentinas: investigaciones y modelos posibles. Los Polvorines: Universidad Nacional de General Sarmiento, EBook.

Fernández Lamarra N. (2002). La Educación Superior en Argentina. Buenos Aires: Eudeba IESALC/Unesco. Imai, M. (1998). Cómo implementar el Kaizen en el sitio de Trabajo (Gemba). Colombia: McGraw-Hill Interamericana de Colombia.

Imai, M. (2001). KAISEN: La clave de la ventaja competitiva japonesa. México: Cecsa.

Hernández Sampieri, R.; Fernández Collado, C. y Baptista Lucio, P. (2006). Metodología de la Investigación. México: McGraw-Hill Interamericana.

León, O. y Montero, I. (2002). Métodos de investigación en psicología y educación. Madrid: McGraw Hill Interamericana de España.

Sandín Esteban, M.P. (2003). Investigación cualitativa en educación: Fundamentos y tradiciones. Madrid: McGraw Hill Interamericana de España.

Tünnermann Bernheim, C. (2000). El nuevo concepto de la extensión universitaria. Universidad Michoacana de San Nicolás de Hidalgo. Morelia, Michoacán, México. Recuperado de http://www.aduba.org.ar/wp-content/ uploads/2016/07/NuevoConceptoExtensionUniversitaria-CarlosTunnermann.pdf 